\title{
A CLUSTER OF MENINGOCOCCAL CASES IN CAMPBELLTOWN
}

$\mathbf{F}$ ifteen cases of meningococcal disease occurred in South Western Sydney Area Health Service (SWSAHS) in 1991. Figure 7 shows the distribution of cases by month, and Table 8 summarises the cases. Seven of these cases (six patients with meningitis and one with septicaemia) occurred between July 17 and August 3. Five of seven patients (cases 5, 6, 8, 10 and 11 of Table 8) were from the Campbelltown Local Government Area (LGA). All the cases from the Campbelltown LGA were caused by Group C meningococcus.

The incidence of meningococcal disease in NSW is about $1-2$ cases $/ 100,000$ people per year ${ }^{1}$. On the basis of this rate, we would expect two or three cases a year of meningococcal disease in the Campbelltown LGA, and 7-13 cases a year in the Area as a whole. While meningococcal disease occurs more commonly in winter and spring ${ }^{2}$, this clustering of cases in late July-early August was a cause of concern, particularly in light of reported outbreaks associated with Group C meningococcus in Western Australia and Queensland ${ }^{3,4}$. The following steps were taken by the SWSAHS Public Health Unit (PHU) in response to this cluster of cases of meningococcal disease:

All isolates were confirmed as Neisseria meningitidis ( $N$. meningitidis) and serogrouping was performed by the Microbiology Department, South Western Area Pathology Service (SWAPS).

The cases were investigated by PHU staff. The admitting hospital arranged rifampicin prophylaxis for family contacts. The PHU assessed whether other contacts required prophylaxis. Associations between the cases were sought. No contact between them could be established. Cases 10 and 11 attended the same school at Campbelltown, but were in different classes and did not play together inside or outside school.

Hospitals in the Area were notified of the cluster to raise awareness among Emergency Department staff of the possibility of meningococcal disease in children presenting with symptoms including fever, vomiting and poor feeding

General practitioners were advised through the Area GP newsletter, prepared by the SWSAHS Division of General Practice and issued in early August.

- Local media were informed and the two Campbelltown newspapers ran stories which explained the need for early medical assessment if a child became unwell.

- A decision was made to offer meningococcal vaccine to all children at the school in which the two cases had occurred, their siblings aged twofive years and children from two local preschools. A few older siblings of children at the school attended a different primary school and were also offered immunisation.

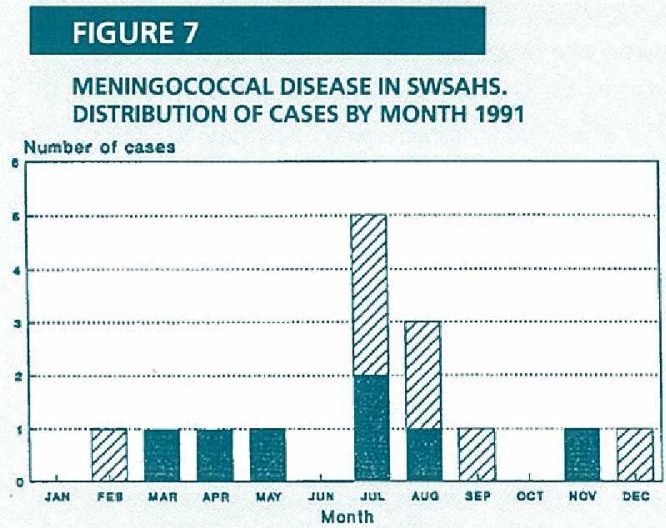

\begin{tabular}{|c|c|c|c|c|c|}
\hline \multicolumn{6}{|c|}{ TABLE 8} \\
\hline \multicolumn{6}{|c|}{$\begin{array}{l}\text { SUMMARY OF CASES OF MENINGOCCAL DISEASE OCCURRING IN } \\
\text { SWSAHS in } 1991\end{array}$} \\
\hline $\begin{array}{l}\text { Case } \\
\text { No. }\end{array}$ & Sex & Age & $\begin{array}{c}\text { Date of } \\
\text { admission }\end{array}$ & LGA & $\begin{array}{c}\text { Lab } \\
\text { Confirmed/ } \\
\text { Group }\end{array}$ \\
\hline 1 & $M$ & 16 yrs & $7 / 2 / 91$ & Campbelltown & Yes Group C \\
\hline 2 & M & $26 \mathrm{mth}$ & $23 / 3 / 91$ & Bankstown & Yes Group C \\
\hline 3 & $\mathrm{~F}$ & $8 \mathrm{mth}$ & $20 / 4 / 91$ & Liverpool & Yes Group B \\
\hline 4 & $\mathrm{~F}$ & $5 \mathrm{mth}$ & $26 / 5 / 91$ & Fairfield & Yes Group C \\
\hline 5 & $\mathrm{~F}$ & 2.5 yrs & $17 / 7 / 91$ & Campbelltown & Yes Group C \\
\hline 6 & $\mathrm{~F}$ & 14 yrs & $18 / 7 / 91$ & Campbelltown & Yes Group C \\
\hline 7 & M & $16.5 \mathrm{yrs}$ & $22 / 7 / 91$ & Fairfield & No \\
\hline 8 & M & 5 mth & $28 / 7 / 91$ & Campbelltown & Yes Group C \\
\hline 9 & M & 2.5 yrs & $31 / 7 / 91$ & Fairfield & Yes Group B \\
\hline 10 & $\mathrm{~F}$ & 6.5 yrs & $1 / 8 / 91$ & Campbelltown & Yes Group C \\
\hline 11 & M & $10 \mathrm{yrs}$ & $3 / 8 / 91$ & Campbelltown & Yes Group C \\
\hline 12 & M & 5 yrs & $20 / 8 / 91$ & Camden & Yes Group C \\
\hline 13 & M & $11.5 \mathrm{yrs}$ & $12 / 9 / 91$ & Campbelltown & Yes Group C \\
\hline 14 & $\mathrm{~F}$ & 21 yrs & $29 / 11 / 91$ & Camden & Yes Group C \\
\hline 15 & M & 7 mth & $31 / 12 / 91$ & Campbelltown & Yes Group B \\
\hline
\end{tabular}

\section{THE IMMUNISATION CAMPAIGN}

After the decision to conduct an immunisation campaign was made, letters were sent to about 100 local GPs outlining the reasons for the campaign and for targeting this group of children. An information sheet on meningococcal disease (originally prepared by the Western Australian Department of Health and used with its permission) was attached. This strategy had two benefits: it raised GP awareness about meningococcal disease, and it also allowed GPs to provide more complete advice to any parents who sought information.

An explanatory letter, with attached consent for immunisation, was sent to parents of all children at the school and preschools. Letters and consents were translated into Cambodian, Vietnamese, Spanish and Arabic.

The PHU established strong links with the executive of the school and the Primary Health Nurse attached to the school. PHU staff addressed the teachers about meningitis and meningococcal disease and the reasons for the immunisation campaign. Questions about the risk to themselves and their children were discussed, as were organisational matters about the campaign. 


\section{Meningococcal cases in Campbellitown}

\section{- Continued from page 93}

Contact was made with two nearby schools to allay anxiety about the cluster and to give teachers information about meningitis.

The immunisation campaign was conducted in the school auditorium on August 13 and 14. The PHU enlisted the assistance of a range of health care workers from within SWSAHS. PHU staff, Primary Health Nurses, Infection Control staff, and staff from the Microbiology Department of SWAPS assisted in the campaign. Two small catch-up clinics were conducted the following week. A GP with a practice close to the school also assisted by immunising a few children unable to attend the clinics. Vaccine was provided by the PHU.

A total of 892 children was immunised during the campaign. Of these, 646 were school children. This represented an 89 per cent immunisation rate among the school children. As part of the campaign, parents were asked to consent to their child having a throat swab to assess carriage rates of meningococci in the school. Seven hundred and ninety-three swabs were collected. The results of this study will be reported elsewhere.

A follow-up survey assessed side-effects of the vaccine. Among school children, there was a 51 per cent response rate ( 332 out of 646 children immunised) to the survey. No serious adverse effects were reported; 17 per cent reported a sore arm for more than two days, 25 per cent reported redness at the injection site and 12 per cent reported a high temperature after immunisation.

\section{DISCUSSION}

In recent years an increase in meningococcal disease has been observed throughout Australia, with the Group C meningococcus comprising an increasing proportion of $N$. meningitidis isolates. This finding is consistent with the data in SWSAHS, where 11 of the 15 cases of meningococcal disease (73 per cent) occurring between January and December 1991 were caused by Group C meningococci. Three of 15 ( 20 per cent) were caused by Group B meningococci.

Vaccination has been advocated in the control of outbreaks caused by Group $\mathrm{A}$ and $\mathrm{C}$ meningococcus. The vaccine available in Australia (Mencevax AC, supplied by Smith Kline Biologicals) contains purified meningococcal polysaccharides $\mathrm{A}$ and $\mathrm{C}$. A single dose induces protective antibodies within 10-14 days in about 90 per cent of recipients over the age of two years ${ }^{6}$.

Recent outbreaks caused by Group C meningococcus have occurred in Katanning in Western Australia and Doomadgee in Queensland ${ }^{3,4}$. Widespread immunisation campaigns in both cases contained the outbreaks.

The decision was made to conduct the immunisation campaign at the school because a defined group at increased risk could be identified, given that two pupils at the school had developed Group $\mathrm{C}$ meningococcal disease. There was also concern that this cluster of five cases in the Campbelltown LGA occurring within an 18-day period may have represented the beginning of a larger outbreak.
Siblings aged two-five years were offered immunisation because of their close links with the school community, and because children less than five years of age have an increased risk of developing meningococcal disease'. Children at two local preschools were offered immunisation because the majority were siblings of children at the school, and this approach facilitated access to the group. The community was aware that two children at a local school had developed meningococcal disease. This had given rise to concern, particularly among parents of school-aged children. When it became known that an immunisation campaign was being conducted at the school, parents with children at nearby schools contacted the PHU requesting immunisation, or presented for immunisation. For this reason, age and residential criteria for immunisation were established before the campaign. Children had to be aged between two and 12 years, and either attend the school or reside in the Education Department's catchment area for the school. Apart from a handful of special cases (exceptions were determined by the Medical Officer of Health), these criteria were adhered to. This involved explaining to several parents and/or their GPs why immunisation was not recommended for their children.

The decision to immunise was made only after consultation with a range of experts, both in Sydney and interstate, and an extensive review of the literature. The potential avoidance of further cases had to be weighed against the potential side-effects of the vaccine. In addition, the vaccine is expensive and although it was supplied at a discount by Smith Kline Biologicals, the total cost was more than $\$ 23,000$.

The circumstances under which immunisation should be used in the control of meningococcal disease are not clear. The National Health and Medical Research Council Communicable Diseases Standing Committee has decided that a working party should be established to formulate guidelines on the role of immunisation in the management of outbreaks of meningococcal disease. From August 3 until the end of 1991 two further cases of meningococcal disease occurred in the Campbelltown LGA (one Group C, one Group B). No cases occurred in children who had been immunised.

Kerry Chant, Public Health Officer,

SWSAHS Public Health Unit.

Greg Stewart, Medical Officer of Health,

SWSAHS Public Health Unit.

John Brown, Public Health Nurse,

SWSAHS Public Health Unit.

Rosemary Munro, Director of Microbiology,

South Western Area Pathology Service.

George Toouli, Senior Hospital Scientist,

Microbiology Department SWAPS.

Katherine Kociuba, Microbiology Registrar, SWAPS.

1. Levy M, Manning W, Rubin G. Bacterial meningitis makes

a comeback. NSW Public Health Bulletin 1991; 2:8-10.

2. Benenson AS (ed). Control of Communicable Diseases in Man.

14th edition. American Public Health Association. Washington DC. 1985.

3. Watson C, Gardner V. A cluster of cases of Group C meningococcal infection in Katanning, Western Australia. CDI 90/5:4-7.

4. Pearce M Sheridan, An extrardinary putbreak 4. Pearce M, Sheridan J. An extraordinary outbreak of meningococcal meningitis at Doomadgee Aboriginal Community. CDI 91/10:168-169. 5. Clements DA, Gilbert L. Increase in meningococcal infections detected at the Royal Children's Hospital, Melbourne. CDI 90/8:4-7.

6. Hall R. Editorial comment. CDI 90/5:6-7 